\title{
MAXIMAL FUNCTIONS AND SUBORDINATION FOR OPERATOR GROUPS
}

\author{
GORDON BLOWER \\ Department of Mathematics and Statistics, Lancaster University, \\ Lancaster LA1 4YF, UK (g.blower@lancaster.ac.uk)
}

(Received 15 May 2000)

\begin{abstract}
Let $E$ be a UMD Banach space, and $L$ a positive self-adjoint operator in $\mathrm{L}^{2}$ of Laplace type, for which the imaginary powers $L^{-i t}$ form a $C_{0}$-group of exponential growth $0 \leqslant \alpha<\pi$ on $\mathrm{L}^{p}(E)$, where $1<$ $p<\infty$. Suppose $G(z)$ is holomorphic inside and on the boundary of the sector $\{z: z \neq 0,|\arg z| \leqslant \phi\}$, and $z^{\kappa} G(z) \rightarrow 0$ uniformly as $z \rightarrow \infty$ for some $\kappa>0$ and $\phi>\alpha$. Then $G(t L)(t>0)$ defines a bounded family of linear operators on $\mathrm{L}^{p}(E)$; and the maximal operator $f \mapsto \sup _{t>0}\|G(t L) f\|_{E}$ is bounded on the domain of $\log L$. The proof uses transference methods. These hypotheses hold for the maximal solution operators for the heat, wave and Schrödinger equations, and for Cesàro sums.
\end{abstract}

Keywords: maximal functions; transference; UMD Banach spaces

AMS 2000 Mathematics subject classification: Primary 47D03; 42B25; 47D09

\section{Introduction and main results}

Let $X$ be a smooth manifold, and $\mu$ a $\sigma$-finite positive Radon measure on $X$. Suppose that $L$ is an operator of Laplace type over $X$, so that $L$ defines a positive and selfadjoint operator in a dense linear subspace of $\mathrm{L}^{2}(\mu)$ with core the smooth functions of compact support $C_{\mathrm{c}}^{\infty}(X)$; to avoid possible ambiguity, we further suppose that the spectral projection corresponding to the singleton set $\{0\}$ is zero. Any bounded and Borel measurable function $G:(0, \infty) \rightarrow \mathbb{C}$ gives rise to a bounded linear operator $G(L)$ on $\mathrm{L}^{2}(\mu)$ by the spectral theorem.

The solutions to the abstract Cauchy problem $\partial u / \partial t=\mathrm{i} L u, u(0, x)=f(x)$, for the Schrödinger equation are given by $u(t, x)=\mathrm{e}^{\mathrm{i} t L} f(x)$; although this problem is well posed for initial data $f \in \mathrm{L}^{2}(\mu)$, one typically obtains $\mu$-almost sure convergence to the initial data, $u(t, x) \rightarrow f(x)$ as $t \rightarrow 0$, only for $f$ in a proper subspace of $\mathrm{L}^{2}(\mu)[\mathbf{1 3}, \mathbf{1 7}]$. In $[\mathbf{5}, \mathbf{9}]$ a technique for proving such results was introduced, which was based on analysis of the maximal operators $f \mapsto \sup _{t>0}|G(t L) f(x)|$. Using the Mellin transform of $G$, one can reduce the analysis of the maximal operator to the group $L^{\mathrm{i} v}$ of imaginary powers of $L$. Typically this defines a $C_{0}$-group on $\mathrm{L}^{p}(\mu)(1<p<\infty)$, which is not uniformly bounded for $p \neq 2$ [18]. In this paper we achieve new maximal theorems for operator families defined using such groups. To obtain estimates on various operators by transference 
methods, it is necessary to restrict attention to a special class of Banach spaces which includes $\mathrm{L}^{p}(\mu)(1<p<\infty)$.

Definition 1.1. For $E$ a complex Banach space, the Bochner-Lebesgue space $\mathrm{L}^{p}(\mu ; E)$ consists of strongly measurable $E$-valued functions $f$ on $X$ for which the norm

$$
\|f\|_{\mathrm{L}^{p}(\mu ; E)}=\left(\int_{X}\|f(s)\|_{E}^{p} \mu(\mathrm{d} s)\right)^{1 / p}
$$

is finite. And $E$ is said to be a UMD space if the Hilbert transform

$$
H(f)(t)=\mathrm{PV} \int_{-\infty}^{\infty} f(t-s) \frac{\mathrm{d} s}{\pi s} \quad\left(f \in \mathrm{L}^{p}(\mathbb{R} ; E)\right)
$$

defines a bounded linear operator on $\mathrm{L}^{p}(\mathbb{R} ; E)$ for $1<p<\infty$. See $[\mathbf{3}, \mathbf{6}, \mathbf{7}]$ for examples.

When $E$ is a UMD Banach space, one seeks to extend the definition of $L^{\mathrm{i} v}$ to $L^{\mathrm{i} v} \otimes \operatorname{Id}_{E}$ on $\mathrm{L}^{p}(\mu ; E)$ (where $\operatorname{Id}_{E}$ denotes the identity operator on $E$ ) by using the functional calculus of spectral integration $[\mathbf{2}, \mathbf{3}]$; for notational simplicity, one then writes $L^{\mathrm{i} v}$ for this extended operator. The domain of the generator i $\log L$ of the group $L^{\mathrm{i} u}$ in $\mathrm{L}^{p}(\mu ; E)$ has the usual graph norm

$$
\|f\|_{\mathbb{D}(\log L)}=\|f\|_{\mathrm{L}^{p}(\mu ; E)}+\|(\log L) f\|_{\mathrm{L}^{p}(\mu ; E)} .
$$

Using a much improved version of the technique of [5], we obtain results for maximal functions associated with operator families $G(t L)$ on $\mathrm{L}^{p}(\mu ; E)$ in $\S 2$. Our first application, Theorem 3.1, relates to the maximal operator for the holomorphic extension of the heat semigroup $H_{t}=\mathrm{e}^{-t L}$, when $L^{\mathrm{i} v}$ is of exponential growth of rate less than $\pi / 2$. In $\S 3$ we also obtain, under stronger hypotheses, bounds on the solution operator for the wave and Schrödinger equations. Our main result is the following theorem.

Theorem 1.2. Suppose that $L^{\mathrm{i} v}(v \in \mathbb{R})$ defines a $C_{0}$-group on $\mathrm{L}^{p}(\mu ; E)$ for some $1<p<\infty$ and UMD Banach space $E$, with

$$
\left\|L^{\mathrm{i} v}\right\|_{B\left(\mathrm{~L}^{p}(\mu ; E)\right)} \leqslant C\left(1+|v|^{\beta}\right) \quad(v \in \mathbb{R}),
$$

where $\beta<\frac{1}{2}$. Then

(a) the wave sine family $\sin (t \sqrt{L}) / t \sqrt{L}(t>0)$ defines a uniformly bounded family of linear operators on $\mathrm{L}^{p}(\mu ; E)$, and the sublinear maximal operator $W_{*}: \mathbb{D}_{\mathrm{L}^{p}(\mu ; E)}(\log L) \rightarrow \mathrm{L}^{p}(\mu)$ is bounded, where

$$
W_{*}: f \mapsto \sup _{t>0}\left\|\frac{\sin (t \sqrt{L})}{t \sqrt{L}} f(x)\right\|_{E} \quad\left(f \in \mathbb{D}_{\mathrm{L}^{p}(\mu ; E)}(\log L)\right) ;
$$

and

(b) likewise, the family of Schrödinger sine operators $\sin (t L) / t L(t>0)$ is uniformly bounded on $\mathrm{L}^{p}(\mu ; E)$ and the sublinear maximal operator $S_{*}: \mathbb{D}_{\mathrm{L}^{p}(\mu ; E)}(\log L) \rightarrow$ $\mathrm{L}^{p}(\mu)$ is bounded, where

$$
S_{*}: f \mapsto \sup _{t>0}\left\|\frac{\sin (t L)}{t L} f(x)\right\|_{E}\left(f \in \mathbb{D}_{\mathrm{L}^{p}(\mu ; E)}(\log L)\right) .
$$


In $\S 4$ we derive some consequences for the Euclidean Laplace operator.

We shall show that the maximal operator $W_{*}$ of (1.3) has similar properties to the Hardy-Littlewood maximal operator. The relations between the various maximal operators are discussed in $\S 6$.

Our main 'Maximal Lemma' 2.3 also has consequences for Cesàro summability of orthogonal series. Let $\left(\varphi_{n}\right)(n \in \mathbb{N})$ be an orthonormal basis of $\mathrm{L}^{2}(\mu)$ for some probability measure on $X$, and for $f \in \mathrm{L}^{2}(\mu)$ write $f \sim \sum_{n} a_{n} \varphi_{n}$ for the orthogonal series generated by $f$. For a real sequence of $\lambda_{n} \geqslant 1$ with $\lambda_{n} \rightarrow \infty$ as $n \rightarrow \infty$, we can introduce a selfadjoint and positive operator in $\mathrm{L}^{2}(\mu)$ by setting $L \varphi_{n}=\lambda_{n} \varphi_{n}$ and extending linearly. In $\S 5$ we prove results including the following theorem.

Theorem 1.3. Suppose $\delta>0$ and that $f$ belongs to the domain of $\log L$, so

$$
\sum_{n}\left(1+\log \lambda_{n}\right)^{2}\left|a_{n}\right|^{2}<\infty .
$$

Then $\sum_{n} a_{n} \varphi_{n}(x)$ is almost everywhere $(C, \delta)$ summable to $f(x)$, so

$$
\sum_{n: \lambda_{n} \leqslant N}\left(1-\frac{\lambda_{n}}{N}\right)^{\delta} a_{n} \varphi_{n}(x) \rightarrow f(x),
$$

$\mu$-almost everywhere as $N \rightarrow \infty$.

In this context, it is natural to have maximal operators defined only on the domain of $\log L$, as illustrated by the theorem of Menshov [16]:

Given $\varepsilon>0$, there exists an orthonormal basis $\left(\varphi_{n}\right)(n \in \mathbb{N})$ of $\mathrm{L}^{2}[0,1]$ for which the maximal partial sum operator

$$
\sum_{n} a_{n} \varphi_{n} \mapsto \sup _{N(x)}\left|\sum_{n=1}^{N(x)} a_{n} \varphi_{n}(x)\right|
$$

is unbounded $\mathbb{D}_{\mathrm{L}^{2}}\left((\log L)^{1-\varepsilon}\right) \rightarrow \mathrm{L}^{1}[0,1]$ where $L$ is determined by $\lambda_{n}=n+1$ $(n \in \mathbb{N})$.

The proof of the main 'Maximal Lemma' in $\S 2$ involves a functional calculus argument, which the reader may find more congenial when expressed in the language of Fourier transforms. For $\kappa, \beta>0$, let $\mathbb{A}_{\beta, \kappa}$ be the space of holomorphic functions $g(z)$ on the strip $\Omega_{\beta}=\{z:|\operatorname{Im} z|<\beta\}$ such that $(\mathrm{a}) \mathrm{e}^{\kappa z} g(z)$ and $(\mathrm{b}) \mathrm{e}^{-\kappa z} g^{\prime}(z)$ are bounded on $\Omega_{\beta}$. It is easy to verify that any such $g$ is itself bounded on $\Omega_{\beta}$, and hence $\mathbb{A}_{\beta, \kappa}$ forms a Banach algebra under pointwise multiplication for the norm

$$
\|g\|_{\mathbb{A}_{\beta, \kappa}}=\sup _{z \in \Omega_{\beta}}\{|g(z)|\}+\sup _{z \in \Omega_{\beta}}\left\{\left|\mathrm{e}^{\kappa z} g(z)\right|\right\}+\sup _{z \in \Omega_{\beta}}\left\{\left|\mathrm{e}^{-\kappa z} g^{\prime}(z)\right|\right\} .
$$

Theorem 1.4. Let $E$ be a UMD Banach space, and $T_{t}=\mathrm{e}^{\mathrm{i} t A}$ a $C_{0}$-group of operators on $E$, with $\left\|T_{t}\right\|_{B(E)} \leqslant M \mathrm{e}^{\alpha|t|}(t \in \mathbb{R})$. Then, for any $\beta>\alpha$ and $\kappa>0$, there is a bounded homomorphism $\mathbb{A}_{\beta, \kappa} \rightarrow B(E): g \mapsto g(A)$ for any $\kappa>0$. 
The main idea in the proof is that

$$
\hat{g}(u+\mathrm{i} v)=\int_{-\infty}^{\infty} g(x+\mathrm{i} y) \mathrm{e}^{-\mathrm{i}(x+\mathrm{i} y)(u+\mathrm{i} v)} \mathrm{d} x
$$

is independent of $-\beta<y<\beta$ by Cauchy's Theorem, and defines a holomorphic function on $\{u+\mathrm{i} v: 0<v<\kappa,-\infty<u<\infty\}$. Using (b) and an integration by parts argument, one can extend $\hat{g}$ to a meromorphic function on $\Omega_{\kappa}$ for which the only possible singularity is a simple pole at zero. Further, for any $\beta^{\prime}<\beta$ and $\kappa^{\prime}<\kappa$, the extended function $\hat{g}(u+\mathrm{i} v)$ decays at an exponential rate $\beta^{\prime}$ uniformly on $\Omega_{\kappa^{\prime}}$ as $u \rightarrow \pm \infty$.

The functional calculus map is

$$
g \mapsto \lim _{\varepsilon \rightarrow 0+} \frac{1}{2 \pi}\left(\int_{-\infty}^{-\varepsilon}+\int_{\varepsilon}^{\infty}\right) \hat{g}(u) T_{u} \mathrm{~d} u-\frac{1}{2} \mathrm{i} \operatorname{Res}(\hat{g} ; 0) \operatorname{Id}_{E},
$$

wherein the integrals converge at infinity on account of the exponential decay of the Fourier transform, while the singularity at zero may be treated by transference methods.

This provides us with one particular way of defining $g(A)$ when i $A$ generates a bounded $C_{0}$-group of operators, and $g$ lies in the proper subalgebra $\mathbb{A}_{\beta, \kappa}$ of the usual Hardy space $\mathrm{H}^{\infty}\left(\Omega_{\beta}\right)$ of bounded holomorphic functions on some fixed $\Omega_{\beta}$. For $\alpha, s, \varepsilon>0$ with $2 \alpha s>\kappa$ and $\alpha \beta<\pi / 2$, the function $m_{\alpha, s}(z)=(\operatorname{sech}(\alpha z))^{2 s}$ multiplies $f \in \mathrm{H}^{\infty}\left(\Omega_{\beta+\varepsilon}\right)$ pointwise to an element $m_{\alpha, s} f$ of $\mathbb{A}_{\beta, \kappa}$. By introducing $L=\mathrm{e}^{-A}$ one can work with bounded holomorphic functions on sectors instead of strips. In [10] the authors develop an $\mathrm{H}^{\infty}$ functional calculus for operators $L$ of type $\omega$, such that $\left\|z(L-z I)^{-1}\right\| \leqslant M_{\theta}$ whenever $|\arg z|>\theta>\omega$, for which the imaginary powers generate $C_{0}$-groups with $\left\|L^{\mathrm{is}}\right\| \leqslant M_{\mu} \mathrm{e}^{\mu|s|}(s \in \mathbb{R})$ for $\omega<\mu<\pi$. Their Convergence Lemma 2.1 shows that the $\mathrm{H}^{\infty}$ functional calculus on $\{z: z \neq 0,|\arg z| \leqslant \mu\}$ is unique under mild hypotheses. This holds for general Banach spaces, and some of their arguments simplify in the context of the present paper, namely UMD Banach spaces which are reflexive. Certain functional calculus results of $\S 2$ below are essentially contained in $[\mathbf{1 0}]$, wherein the function $z^{s} /(1+z)^{2 s}$ corresponds to $m_{(1 / 2), s}$.

Nevertheless, Lemma 5.2 in $[\mathbf{1 0}]$ shows that on $\mathrm{L}^{p}(\mathbb{R})$ with $p \neq 2$ one can construct a densely defined operator $L$ for which $L^{\text {iu }}$ defines a bounded $C_{0}$-group, but for which $L$ does not admit a bounded functional calculus for $\mathrm{H}^{\infty}$ functions on any sector $\{z: z \neq 0,|z| \leqslant \phi\}$ with $0<\phi<\pi$. The functional calculus of [3, Theorem 5.6] provides an alternative method for bounding functions of $L$, and the spectral family of projections of $[\mathbf{3}$, Theorem 5.1$]$ is essentially unique.

\section{Maximal Lemma for imaginary powers}

Throughout this section, we suppose $1<p<\infty$, and $E$ is a UMD Banach space. We also suppose that the group of imaginary powers $L^{\text {iv }}(v \in \mathbb{R})$ defines a $C_{0}$-group on $\mathrm{L}^{p}(\mu ; E)$ which satisfies

$$
\left\|L^{\mathrm{i} v}\right\|_{B\left(\mathrm{~L}^{p}(\mu ; E)\right)} \leqslant M_{L} \mathrm{e}^{\alpha|v|} \quad(v \in \mathbb{R}) .
$$


Stein $[\mathbf{1 8}$, p. 58] shows that the negative generator of any symmetric diffusion semigroup $\mathrm{e}^{-t L}$ satisfies (2.1) with $\alpha \leqslant \pi / 2$ when $E=\mathbb{C}$; this gives us our main source of examples (see also $[4,5.2]$ ).

Using the Mellin transform, we shall show that $G(L) \otimes \operatorname{Id}_{E}$ may be extended from $C_{\mathrm{c}}^{\infty}(X) \otimes E$ to define a bounded linear operator $G(L)$ on $\mathrm{L}^{p}(\mu ; E)$, provided $G$ satisfies various technical conditions. We first impose a strong hypothesis, to be weakened later:

(i) $G(x) / x^{1+\gamma}$ is integrable over $x$ in $(0, \infty)$ for $-\delta<\gamma<\delta$, for some $\delta>0$.

The Mellin transform may then be defined by

$$
\tilde{G}(s)=\int_{0}^{\infty} x^{s-1} G(x) \mathrm{d} x,
$$

where the integral is absolutely convergent and is a holomorphic function of $s$ on the strip $\{s:-\delta<\operatorname{Re} s<\delta\}$. The Mellin inversion formula [21, p. 46] gives

$$
G(\lambda)=\lim _{R \rightarrow \infty} \frac{1}{2 \pi \mathrm{i}} \int_{\gamma-\mathrm{i} R}^{\gamma+\mathrm{i} R} \tilde{G}(s) \lambda^{-s} \mathrm{~d} s \quad(-\delta<\gamma=\operatorname{Re} s<\delta)
$$

at all points of differentiability of $G$. Similarly, if $\tilde{G}(\gamma+\mathrm{i} v)$ is integrable over $v \in(-\infty, \infty)$ and differentiable at $\gamma+\mathrm{it}$, then

$$
G(x)=\frac{1}{2 \pi \mathrm{i}} \int_{\gamma-\mathrm{i} \infty}^{\gamma+\mathrm{i} \infty} \tilde{G}(s) x^{-s} \mathrm{~d} s
$$

satisfies

$$
\tilde{G}(\gamma+\mathrm{i} t)=\lim _{\substack{\eta \rightarrow 0+\\ R \rightarrow \infty}} \int_{\eta}^{R} x^{\gamma+\mathrm{i} t-1} G(x) \mathrm{d} x .
$$

For $0<\phi<\pi$ we introduce the sector $K_{\phi}=\{z: z \neq 0,|\arg z| \leqslant \phi\}$ and consider the complex functions $G$ which are continuous on $K_{\phi}$ and holomorphic on its interior. These assumptions will be in force throughout this section.

We recall the proof of a fundamental result $[\mathbf{5}, \mathbf{9}]$, in which we further suppose that

(ii) $G(z) \rightarrow 0$ as $|z| \rightarrow 0$ and as $|z| \rightarrow \infty$ in $K_{\phi}$, uniformly in $|\arg z| \leqslant \phi$; and

(iii) $r^{-1} G\left(r \mathrm{e}^{ \pm \mathrm{i} \phi}\right)$ are integrable over $r \in(0, \infty)$.

Proposition 2.1. Suppose that $G$ satisfies (i), (ii) and (iii) for some $\pi>\phi>\alpha$. Then $G(t L)(t>0)$ defines a uniformly bounded family of operators on $\mathrm{L}^{p}(\mu ; E)$, and the maximal operator $f \mapsto \sup _{t>0}\|G(t L) f(x)\|_{E}$ defines a bounded sublinear operator $\mathrm{L}^{p}(\mu ; E) \rightarrow \mathrm{L}^{p}(\mu)$.

Proof. Using Cauchy's Theorem and condition (ii), we can turn the line of integration in the definition of the Mellin transform to $\arg z=\operatorname{sgn}(v) \phi=\psi$ and thereby achieve

$$
\tilde{G}(\mathrm{i} v)=\mathrm{e}^{-|v| \phi+\mathrm{i} \psi} \int_{0}^{\infty} r^{\mathrm{i} v-1} G\left(r \mathrm{e}^{\mathrm{i} \psi}\right) \mathrm{d} r \quad(v \in \mathbb{R}) .
$$


By the inversion formula, we can then write

$$
G(t L) f=\frac{1}{2 \pi} \int_{-\infty}^{\infty} \tilde{G}(\mathrm{i} v) t^{-\mathrm{i} v} L^{-\mathrm{i} v} f \mathrm{~d} v \quad\left(f \in \mathrm{L}^{p}(\mu ; E), t>0\right) .
$$

For any $x \in X$, we have by the triangle inequality

$$
\sup _{t>0}\|G(t L) f(x)\|_{E} \leqslant \frac{1}{2 \pi} \int_{-\infty}^{\infty}|\tilde{G}(\mathrm{i} v)|\left\|L^{-\mathrm{i} v} f(x)\right\|_{E} \mathrm{~d} v,
$$

since $\left|t^{-\mathrm{i} v}\right|=1$. Taking the $\mathrm{L}^{p}(\mu)$ norm of both sides gives

$$
\begin{aligned}
\left\|\sup _{t>0}\right\| G(t L) f\left\|_{E}\right\|_{L^{p}(\mu)} & \leqslant \frac{1}{2 \pi} \int_{-\infty}^{\infty}|\tilde{G}(\mathrm{i} v)|\left\|L^{-\mathrm{i} v} f\right\|_{\mathrm{L}^{p}(\mu ; E)} \mathrm{d} v \\
& \leqslant \frac{1}{2 \pi} \int_{-\infty}^{\infty}|\tilde{G}(\mathrm{i} v)|\left\|L^{-\mathrm{i} v}\right\|_{B\left(\mathrm{~L}^{p}(\mu ; E)\right)} \mathrm{d} v \times\|f\|_{\mathrm{L}^{p}(\mu ; E)},
\end{aligned}
$$

for any $f \in \mathrm{L}^{p}(\mu ; E)$; and the latest integral is convergent, since the exponential decay rate of the Mellin transform exceeds the growth rate of the group by (2.3) and (iii).

Unfortunately, neither condition (i) nor (ii) is satisfied in many cases of interest, such as Theorems 1.2, 3.1 and parts (a) and (b) of Corollary 4.1, wherein the Mellin transform $\tilde{G}(s)$ has a simple pole at $s=0$. Consequently, the constant $C(p, \alpha)$ of Cowling's fundamental inequality $[\mathbf{9}$, p. 276] becomes infinite, and one cannot control (the maximal function of) the family $G(t L)(t>0)$ by a simple application of the triangle inequality. The main technical contribution of the present paper is to use transference methods to overcome this difficulty. This involves restricting the range of values of $p$, restricting the class of Banach spaces and defining the maximal operator on a proper subspace of $\mathrm{L}^{p}(\mu ; E)$.

Lemma 2.2. Suppose that for some $\kappa>0$ and $0<\phi<\pi$ :

(iv) $G(z)$ is holomorphic with continuous derivative inside and on the boundary of $K_{\phi}$, including $z=0$; and

(v) $G\left(r \mathrm{e}^{\mathrm{i} \theta}\right) r^{\kappa} \rightarrow 0$ as $r \rightarrow \infty$, uniformly for $|\theta| \leqslant \phi$.

Then $\tilde{G}(s)$ extends to define a holomorphic function on the strip $\{s:-1<\operatorname{Re} s<\kappa\}$, apart, possibly, from a simple pole at $s=0$. Furthermore, $\tilde{G}(s)$ decays to zero at exponential rate $\phi$, uniformly for $-\frac{1}{2} \leqslant \operatorname{Re} s \leqslant \frac{1}{2} \kappa$ as $\operatorname{Im} s \rightarrow \pm \infty$.

Proof. For $0<\operatorname{Re} s<\kappa$, the Mellin transform integral is absolutely convergent and defines a holomorphic function on this strip by Morera's Theorem. By applying Cauchy's Theorem to $z^{s-1} G(z)$ on the contour made up of the straight line segments $[\delta, R],\left[\delta \mathrm{e}^{\mathrm{i} \theta}, R \mathrm{e}^{\mathrm{i} \theta}\right]$ and the circular arcs joining $\delta$ to $\delta \mathrm{e}^{\mathrm{i} \theta}$ and $R$ to $R \mathrm{e}^{\mathrm{i} \theta}$, respectively, we can turn the line of integration to $\arg z=\theta$ for any $-\phi \leqslant \theta \leqslant \phi$. Letting $\delta \rightarrow 0+$ and 
$R \rightarrow \infty$, we derive the identity

$$
\begin{array}{r}
\tilde{G}(s)=\mathrm{e}^{\mathrm{i} \theta s}\left(\frac{G\left(\mathrm{e}^{\mathrm{i} \theta}\right)}{s}-\frac{1}{s} \int_{0}^{1} r^{s} G^{\prime}\left(r \mathrm{e}^{\mathrm{i} \theta}\right) \mathrm{d} r+\int_{1}^{\infty} r^{s-1} G\left(r \mathrm{e}^{\mathrm{i} \theta}\right) \mathrm{d} r\right) \\
(|\theta| \leqslant \phi, 0<\operatorname{Re} s<\kappa)
\end{array}
$$

by splitting the range of integration and integrating by parts.

One can use the right-hand side of $(2.7)$ to continue $\tilde{G}(s)$ across the imaginary axis to a meromorphic function on $\{s:-1<\operatorname{Re} s<\kappa\}$, for which the only possible singularity is a simple pole at $s=0$. On account of the initial exponential factor, it also follows that $|\tilde{G}(u+\mathrm{i} v)| \leqslant C_{G} \mathrm{e}^{-\phi|v|}$ for $-\frac{1}{2} \leqslant u \leqslant \frac{1}{2} \kappa$ and $|v| \geqslant 1$ for some $C_{G}<\infty$.

Lemma 2.3 (Maximal Lemma). Let $G$ be as in Lemma 2.2, and suppose that $L^{\mathrm{i} u}$ satisfies (2.1), for some $\pi>\phi>\alpha$. Then $G(t L)(t>0)$ defines a uniformly bounded family of linear operators on $\mathrm{L}^{p}(\mu ; E)$; furthermore, the maximal operator $f \mapsto \sup _{t>0}\|G(t L) f\|_{E}$ defines a bounded sublinear operator $\mathbb{D}_{\mathrm{L}^{p}(\mu ; E)}(\log L) \rightarrow \mathrm{L}^{p}(\mu)$.

Proof. For $\eta>0$ sufficiently small, the Mellin inversion formula may be applied to $\tilde{G}(s+\eta)$, the Mellin transform of $x^{\eta} G(x)$. One can then use Cauchy's theorem to deform the line of integration into a curve avoiding $s=0$. On letting $\eta \rightarrow 0$, this contour may in turn be replaced by the sum of a principal-value integral and the contribution of the pole at zero. Consequently, for $f \in \mathrm{L}^{p}(\mu ; E)$ and $t>0$ we can use the Mellin inversion formula to write

$$
\begin{aligned}
G(t L) f=\frac{1}{2 \pi}\left(\int_{1}^{\infty}+\int_{-\infty}^{1}\right) & \tilde{G}(\mathrm{i} v) t^{-\mathrm{i} v} L^{-\mathrm{i} v} f \mathrm{~d} v \\
& +\operatorname{PV} \frac{1}{2 \pi} \int_{-1}^{1} \tilde{G}(\mathrm{i} v) t^{-\mathrm{i} v} L^{-\mathrm{i} v} f \mathrm{~d} v+2^{-1} \operatorname{Res}(\tilde{G} ; 0) f .
\end{aligned}
$$

The integrals over $(1, \infty)$ and $(-\infty,-1)$ may be bounded by the argument of Proposition 2.1, whereas the final integral in (2.8) requires a more subtle argument: starting with the decomposition $\tilde{G}(\mathrm{i} v)=b / \mathrm{i} v+h(v)$, where $b$ is a constant and $h(v)$ is continuous near to the simple pole at $v=0$, we have an identity of principal-value integrals

$$
\int_{-1}^{1} \tilde{G}(\mathrm{i} v) t^{-\mathrm{i} v} L^{-\mathrm{i} v} f \mathrm{~d} v=\int_{-1}^{1} h(v) t^{-\mathrm{i} v} L^{-\mathrm{i} v} f \mathrm{~d} v+b \int_{-1}^{1}(t L)^{-\mathrm{i} v} f \frac{\mathrm{d} v}{\mathrm{i} v} .
$$

The first of these integrals is clearly bounded with bound independent of $t$, as in Proposition 2.1. The last is the transferred finite Hilbert transform for the representation $v \mapsto(t L)^{-\mathrm{i} v}$ of $\mathbb{R}$ on $\mathrm{L}^{p}(\mu ; E)$, and by $[\mathbf{3}]$ and $[\mathbf{4}$, Theorem 4.1$]$ this operator is bounded with bound independent of $t$. Together these estimates show that $G(t L)(t>0)$ defines a uniformly bounded family of linear operators. 
To control the associated maximal operator, we take $f \in \mathbb{D}_{\mathrm{L}^{p}(\mu ; E)}(\log L)$ and write the last integral in (2.8) as a sum of principal-value integrals

$$
\begin{array}{r}
\int_{-1}^{1} \tilde{G}(\mathrm{i} v) t^{-\mathrm{i} v} L^{-\mathrm{i} v} f \mathrm{~d} v=\int_{-1}^{1} h(v) t^{-\mathrm{i} v} L^{-\mathrm{i} v} f \mathrm{~d} v+\int_{-1}^{1} \frac{t^{-\mathrm{i} v}-1}{\mathrm{i} v}\left(L^{-\mathrm{i} v}-I\right) f \mathrm{~d} v \\
+\int_{-1}^{1} \frac{t^{-\mathrm{i} v}}{\mathrm{i} v} f \mathrm{~d} v+\int_{-1}^{1} L^{-\mathrm{i} v} f \frac{\mathrm{d} v}{\mathrm{i} v}-\int_{-1}^{1} \frac{\mathrm{d} v}{\mathrm{i} v} f .
\end{array}
$$

The first of these integrals may be treated as in Proposition 2.1. The latest integral is zero; the second last is independent of $t>0$ and is the transferred finite Hilbert transform, hence may be controlled as in (2.9); the third last involves $\int_{0}^{1} \sin (v \log t) \mathrm{d} v / v$, which is bounded independently of $t>0$. It remains to control the second integral, which may be written as

$$
\int_{-1}^{1}\left(t^{-\mathrm{i} v}-1\right) \frac{1}{\mathrm{i} v} \int_{0}^{v} L^{-\mathrm{i} w} \mathrm{~d} w(-\mathrm{i} \log L) f \mathrm{~d} v
$$

by definition of the group generator, wherein $\left|t^{-\mathrm{i} v}-1\right| \leqslant 2$ and

$$
\left\|\frac{1}{\mathrm{i} v} \int_{0}^{v} L^{-\mathrm{i} w} \mathrm{~d} w\right\|_{B\left(\mathrm{~L}^{p}(\mu ; E)\right)} \leqslant \frac{M_{L}}{|v|} \int_{0}^{|v|} \mathrm{e}^{w \alpha} \mathrm{d} w \leqslant M_{L} \mathrm{e}^{\alpha} \quad(|v| \leqslant 1)
$$

by (2.1). Consequently, we can take the supremum over $t>0$, and then the $\mathrm{L}^{p}(\mu)$ norm, to obtain

$$
\left\|\sup _{t>0}\right\| \int_{-1}^{1} \frac{t^{-\mathrm{i} v}-1}{\mathrm{i} v}\left(L^{-\mathrm{i} v}-I\right) f \mathrm{~d} v\left\|_{E}\right\|_{\mathrm{L}^{p}(\mu)} \leqslant 2 M_{L} \mathrm{e}^{\alpha}\|(\log L) f\|_{\mathrm{L}^{p}(\mu ; E)} .
$$

Combining all of these estimates gives the required bound on the maximal function.

\section{Heat, wave and Schrödinger maximal theorems}

Our first application of the Maximal Lemma is to the holomorphic semigroup $\mathrm{e}^{-w L}$. This is associated with the heat equation, in the sense that $U(w, x)=\mathrm{e}^{-w L} f(x)$ satisfies $\partial U / \partial w=-L U$ for $w$ in a sector surrounding the semi-axis $\operatorname{Re} w>0$.

Theorem 3.1. Let $L^{\mathrm{i} v}(v \in \mathbb{R})$ be a $C_{0}$-group on $\mathrm{L}^{p}(\mu ; E)$ for some $U M D$ Banach space $E$ and $1<p<\infty$, that satisfies (2.1) with $\alpha<\pi / 2$; and let $0<\psi<\pi / 2-\alpha$. Then $(-L)$ generates a holomorphic semigroup on $\mathrm{L}^{p}(\mu ; E)$, bounded in the sector $\{w:|\arg w| \leqslant \psi\}$; and there exists $C=C(E, p, \psi, \alpha)<\infty$ such that the associated maximal operator satisfies

$$
\left\|\sup _{w:|\arg w| \leqslant \psi}\right\| \mathrm{e}^{-w L} f\left\|_{E}\right\|_{L^{p}(\mu)} \leqslant C M_{L}\left(\|f\|_{L^{p}(\mu ; E)}+\|(\log L) f\|_{L^{p}(\mu ; E)}\right)
$$

for all $f$ in the domain of the group's generator i $\log L$. 
Proof. The existence of such a semigroup was established in [4, Corollary 4.2]. Let $\omega$ have $|\omega|<\psi<\pi / 2-\alpha$, and set $G_{\omega}(z)=\exp \left(-\mathrm{e}^{\mathrm{i} \omega} z\right)$. One can verify (v) with any $\phi<\pi / 2-\psi$, or calculate the Mellin transform explicitly. Indeed $\tilde{G}_{\omega}(s)$ equals $\Gamma(s) \mathrm{e}^{-\mathrm{i} s \omega}$, which satisfies the asymptotic relation

$$
\left|\tilde{G}_{\omega}(\mathrm{i} v)\right| \asymp\left(\frac{2 \pi}{|v|}\right)^{1 / 2} \mathrm{e}^{\omega v} \mathrm{e}^{-\pi|v| / 2} \quad(v \rightarrow \pm \infty)
$$

by Stirling's formula $[\mathbf{2 0}, 4.4 .1]$; hence $\tilde{G}_{\omega}(\mathrm{i} v)$ decays exponentially with rate at least $\pi / 2-\psi>\alpha$ as $v \rightarrow \pm \infty$. Further, $\tilde{G}_{\omega}(s)$ is holomorphic on a strip surrounding the imaginary axis, apart from a simple pole at zero with residue one.

By Lemma 2.3, $\exp \left(-\mathrm{e}^{\mathrm{i} \omega} t L\right)(t>0,|\omega| \leqslant \psi)$ defines a uniformly bounded family of linear operators on $\mathrm{L}^{p}(\mu ; E)$, and the associated maximal operator is bounded from the domain of $\log L$ to $\mathrm{L}^{p}(\mu)$.

\subsection{Proof of Theorem 1.2}

(a) The function $G(x)=\sin (\sqrt{x}) / \sqrt{x}(x>0)$ has Mellin transform

$$
\tilde{G}(s)=-2 \Gamma(2 s-1) \cos (\pi s),
$$

with poles at $s=-n$, with $n \geqslant 0$ an integer [21, p. 204]. Further, by Stirling's formula [20, 4.41], we have the asymptotic relation

$$
\tilde{G}(\mathrm{i} v) \asymp \frac{-\sqrt{\pi}}{|v|^{3 / 2}} \exp \left(-\pi|v|+2 \mathrm{i} v \log \frac{2|v|}{\mathrm{e}}-\frac{3 \pi \mathrm{i} \operatorname{sgn}(v)}{4}\right) \cosh (\pi v) \quad(v \rightarrow \pm \infty),
$$

which is of the order of $|v|^{-3 / 2}$ as $v \rightarrow \pm \infty$. The result follows as in Lemma 2.3.

(b) The proof of Theorem $1.2(\mathrm{~b})$ is similar.

\section{Maximal operators for Euclidean waves and Schrödinger operators}

The Laplace operator

$$
\Delta=-\sum_{j=1}^{d} \frac{\partial^{2}}{\partial x_{j}^{2}}
$$

is essentially self-adjoint on $C_{\mathrm{c}}^{\infty}\left(\mathbb{R}^{d} ; \mathrm{d} x\right)$ and extends naturally to a closed linear operator in $\mathrm{L}^{p}\left(\mathbb{R}^{d} ; \mathrm{d} x\right)$ for $1 \leqslant p<\infty$.

For this operator, d'Alembert's solution of the wave equation gives a representation of the cosine family as averages over the unit sphere with respect to normalized Lebesgue measure, namely

$$
\cos (t \sqrt{\Delta}) f(x)=\int_{[|y|=1]} f(x+t y) \sigma_{d}(\mathrm{~d} y) \quad\left(f \in C_{\mathrm{c}}^{\infty}\left(\mathbb{R}^{d}\right)\right) .
$$


Corollary 4.1. Let $4 d /(2 d+1)<p<4 d /(2 d-1)$. Then

(a) the maximal operator $W_{*}: \mathbb{D}_{\mathrm{L}^{p}}(\log \Delta) \rightarrow \mathrm{L}^{p}\left(\mathbb{R}^{d}\right)$ is bounded, where

$$
W_{*}: f \mapsto \sup _{t>0}\left|\frac{\sin (t \sqrt{\Delta})}{t \sqrt{\Delta}} f(x)\right| ;
$$

(b) similarly, the maximal operator $S_{*}: \mathbb{D}_{\mathrm{L}^{p}}(\log \Delta) \rightarrow \mathrm{L}^{p}\left(\mathbb{R}^{d}\right)$ is bounded, where

$$
S_{*}: f \mapsto \sup _{t>0}\left|\frac{\sin (t \Delta)}{t \Delta} f(x)\right|
$$

and

(c) for $1>\gamma>\max \{(p+2 p d-4 d) / 2 p,(p+4 d-2 p d) / 2 p\}$, the maximal operator

$$
f \mapsto \sup _{t>0}\left|\frac{\cos (t \sqrt{\Delta})-I}{(t \sqrt{\Delta})^{\gamma}} f(x)\right|
$$

is bounded $\mathrm{L}^{p}\left(\mathbb{R}^{d}\right) \rightarrow \mathrm{L}^{p}\left(\mathbb{R}^{d}\right)$.

Proof. (a), (b) Using the Marcinkiewicz Multiplier Theorem and interpolation as in $\left[\mathbf{5}\right.$, Lemma 3.1], one can achieve the bound $\left\|\Delta^{\mathrm{i} v}\right\|_{B\left(\mathrm{~L}^{p}\left(\mathbb{R}^{d}\right)\right)} \leqslant C_{p, d}(1+|v|)^{\beta}$ with $\beta<\frac{1}{2}$ for such $p$ and $d$. Hence the hypotheses of Theorem 1.2 are satisfied.

(c) The Mellin transform of $G(x)=(\cos \sqrt{x}-1) / x^{\gamma / 2}$ is found to be $\tilde{G}(s)=$ $2 \Gamma(2 s-\gamma) \cos \left(\frac{1}{2} \pi(2 s-\gamma)\right)$, which is holomorphic on the strip $-\frac{1}{2}<\operatorname{Re} s<\frac{1}{4}$. This identity may be deduced from the Mellin transform formula for the sine function [21, p. 204] by integration by parts. Furthermore, by Stirling's formula and the bound on the growth of the group, the integral

$$
\int_{-\infty}^{\infty}|\tilde{G}(\mathrm{i} v)|\left\|\Delta^{-\mathrm{i} v}\right\|_{B\left(\mathrm{~L}^{p}\right)} \mathrm{d} v
$$

is convergent; and so we can argue as with (2.6). This may be compared with [5, Theorems 5.1, 5.2] and [9, Corollary 4].

Remarks 4.2. (a) With $d=1$ and $L=\Delta$, it follows from (4.1) that

$$
\frac{\sin (t \sqrt{\Delta})}{t \sqrt{\Delta}} f(x)=\frac{1}{2 t} \int_{-t}^{t} f(x+u) \mathrm{d} u
$$

and the maximal operator $W_{*}$ of (4.2) is simply the Hardy-Littlewood maximal operator [15, p. 237], so Corollary 4.1 (a) may be improved to the statement that $W_{*}$ is bounded on $\mathrm{L}^{p}(\mathbb{R})$ for $1<p<\infty$. However, Theorem 1.2 does not extend to the case of $p=1$. For it is easy to check that

$$
f(x)=\frac{1}{\sqrt{2 \pi}} \int_{-1}^{1} \mathrm{e}^{-(x-y)^{2} / 2} \mathrm{~d} y
$$


is integrable and belongs to $\mathbb{D}(\log \Delta)$ (defined via the Fourier transform) in $\mathrm{L}^{1}(\mathbb{R})$; but the maximal function of $f$ is not integrable.

(b) The maximal operator $f \mapsto \sup _{t>0}\left|\mathrm{e}^{\mathrm{i} t \Delta} f\right|$ is unbounded $\mathrm{L}^{p}(\mathbb{R}) \rightarrow \mathrm{L}_{\text {loc }}^{p}(\mathbb{R})$ for $1 \leqslant p<2[\mathbf{1 3}$, Theorem 2].

The following results improve upon the cases $d=1$ and $d \geqslant 3$ of Corollary 4.1 (b), respectively. Possibly they are known, but I have not been able to locate a reference.

Proposition 4.3. Let $\Delta$ be the Laplacian over the real line. Then the maximal operator $S_{*}$ for the sine family $\sin (t \Delta) / t \Delta$ defines a bounded sublinear operator $\mathrm{L}^{p}(\mathbb{R}) \rightarrow$ $\mathrm{L}^{p}(\mathbb{R})$ for $1<p<\infty$. Likewise, for the Laplacian over the circle, $S_{*}$ is bounded $\mathrm{L}^{p}(\mathbb{T}) \rightarrow \mathrm{L}^{p}(\mathbb{T})$ for $1<p<\infty$.

Proof. We can represent $\Delta$ by the Fourier multiplier $\Delta \mathrm{e}^{\mathrm{i} \xi x}=\xi^{2} \mathrm{e}^{\mathrm{i} \xi x}$, so $S_{*}$ may be viewed as the maximal convolution operator for the kernels $K_{t}(x-y)$ which represent the multipliers $\sin \left(t \xi^{2}\right) / t \xi^{2}$. Indeed, we have $K_{t}(x-y)=F((x-y) / \sqrt{t}) / \sqrt{t}$, where, by Fourier inversion,

$$
F(u)=\int_{-\infty}^{\infty} \frac{\sin \left(\xi^{2}\right)}{\xi^{2}} \mathrm{e}^{\mathrm{i} \xi u} \frac{\mathrm{d} \xi}{2 \pi} \quad(u \in \mathbb{R}) .
$$

By considering the Fresnel integral $F^{\prime \prime}(u)$, one can derive an asymptotic formula for $F$ which implies $|F(u)| \leqslant C /\left(1+u^{2}\right)(u \in \mathbb{R})$ for some $C<\infty$. It follows that the sine family satisfies

$$
\begin{aligned}
\frac{\sin (t \Delta)}{t \Delta} f(x) & =\frac{1}{\sqrt{t}} \int_{-\infty}^{\infty} F\left(\frac{x-y}{\sqrt{t}}\right) f(y) \mathrm{d} y \\
& \leqslant C \int_{-\infty}^{\infty} \frac{\sqrt{t}}{t+(x-y)^{2}}|f(y)| \mathrm{d} y \\
& =C P_{\sqrt{t}}|f|(x)
\end{aligned}
$$

where $P_{t}=\mathrm{e}^{-t \sqrt{\Delta}}$ is the Poisson semigroup for the upper half-plane [15, p. 142]. By the Hardy-Littlewood Maximal Theorem [15, p. 237], the maximal operator $P_{*}: f \mapsto$ $\sup _{t>0}\left|P_{t} f(x)\right|$ defines a bounded sublinear operator $\mathrm{L}^{p}(\mathbb{R}) \rightarrow \mathrm{L}^{p}(\mathbb{R})$ for $1<p<\infty$; and hence, by (4.6), $S_{*}$ is likewise bounded on $\mathrm{L}^{p}(\mathbb{R})$ for all such $p$.

To show that $S_{*}$ is bounded on $\mathrm{L}^{p}(\mathbb{T})$ for $1<p<\infty$, one uses the Poisson summation formula and the previous estimate to show that

$$
\left|\sum_{n}^{\prime} \frac{\sin \left(n^{2} t\right)}{n^{2} t} \mathrm{e}^{\mathrm{i} n(\theta-\phi)}\right| \leqslant \frac{C \sqrt{t}}{t+(\theta-\phi)^{2}}+C \quad(0 \leqslant \theta, \phi \leqslant 2 \pi)
$$

for $0<t<1$. The proof then proceeds as before.

Proposition 4.4. For dimension $d \geqslant 3$ and $d /(d-1)<p \leqslant \infty$ there exists $C_{p, d}<\infty$ such that all $f \in C_{\mathrm{c}}^{\infty}\left(\mathbb{R}^{d}\right)$ satisfy $\left\|S_{*}(f)\right\|_{\mathrm{L}^{p}} \leqslant C_{p, d}\|f\|_{\mathrm{L}^{p}}$. 
Proof. We can use the spectral theorem and the Fourier inversion formula with (4.5) to obtain

$$
\frac{\sin (t \Delta)}{t \Delta} f(x)=\int_{-\infty}^{\infty} F(u) \cos (u \sqrt{t \Delta}) f(x) \mathrm{d} u
$$

for smooth functions of compact support. Since $F$ is integrable, the inequality

$$
S_{*} f(x) \leqslant C \sup _{t>0}|\cos (t \sqrt{\Delta}) f(x)|
$$

follows by convexity. Stein $[\mathbf{1 9}$, p. 518] has shown that the maximal spherical averaging operator $f \mapsto \sup _{t>0}|\cos (t \sqrt{\Delta}) f(x)|$ defines a strongly $(p, p)$ bounded sublinear operator for this restricted range of $p$ and $d$; his proof depends upon Fourier transform restriction phenomena (see also [17]).

\section{Almost everywhere Cesàro summability of orthogonal series}

Throughout this section we let $\mu$ be a probability measure on $X$, and let $\left(\varphi_{n}\right)(n \in \mathbb{N})$ be an orthonormal basis for $\mathrm{L}^{2}(\mu)$. For $f \in \mathrm{L}^{2}(\mu)$ we let $f \sim \sum_{n} a_{n} \varphi_{n}$ be the orthogonal series generated by $f$. For a real sequence $\left(\mu_{n}\right)(n \in \mathbb{N})$ with $\lambda_{n}=\left|\mu_{n}\right| \geqslant 1$ and $\lambda_{n} \rightarrow \infty$ as $n \rightarrow \infty$, we introduce a self-adjoint operator $A$ in $\mathrm{L}^{2}(\mu)$ by setting $A \varphi_{n}=\mu_{n} \varphi_{n}$ and extending linearly. Then $U_{t}=\mathrm{e}^{\mathrm{i} t A}$ defines a $C_{0}$ unitary group on $\mathrm{L}^{2}(\mu)$. We also introduce the positive self-adjoint operator $L$ by $L \varphi_{n}=\lambda_{n} \varphi_{n}$, so $L^{\mathrm{i} u}$ is a $C_{0}$ unitary group on $\mathrm{L}^{2}(\mu)$.

For $\delta>0$, we say that $\sum_{n} a_{n} \varphi_{n}$ is Cesàro $(C, \delta)$ summable to $f$ at $x$, if

$$
\sum_{n: \lambda_{n}<N}\left(1-\frac{\lambda_{n}}{N}\right)^{\delta} a_{n} \varphi_{n}(x) \rightarrow f(x) \quad(N \rightarrow \infty)
$$

(see [12]). Note that each sum on the left-hand side is finite. We write $C_{t}^{\delta} f=(I-t L)_{+}^{\delta} f$ for the expression on the left-hand side, and $C_{*}^{\delta} f(x)=\sup _{t>0}\left|C_{t}^{\delta} f(x)\right|$ for the associated maximal function.

\subsection{Proof of Theorem 1.3}

We shall show that $C_{*}^{\delta}$ is a bounded sublinear operator $\mathbb{D}_{\mathrm{L}^{2}}(\log L) \rightarrow \mathrm{L}^{2}(\mu)$. Almost everywhere convergence of the $(C, \delta)$ Cesàro sums follows by standard and classical arguments. The Mellin transform of $G(x)=(1-x)_{+}^{\delta}$ is

$$
\tilde{G}(s)=\int_{0}^{1}(1-x)^{\delta} x^{s-1} \mathrm{~d} x=\frac{\Gamma(\delta+1) \Gamma(s)}{\Gamma(\delta+1+s)},
$$

which defines a holomorphic function on the strip $R=\{s:-1<\operatorname{Re} s<1\}$, apart from a simple pole at $s=0$. Further, Stirling's formula leads to the estimate $\tilde{G}(s)=O\left(|s|^{-1-\delta}\right)$ uniformly for $s \in R$ as $|s| \rightarrow \infty$. Arguing as in the proof of Lemma 2.3, we deduce that the associated maximal operator $C_{*}^{\delta} f=\sup _{t>0}|G(t L) f|$ is bounded $\mathbb{D}_{\mathrm{L}^{2}}(\log L) \rightarrow \mathrm{L}^{2}(\mu)$. 
Definition 5.1. A bounded linear operator $U$ on $\mathrm{L}^{2}(\mu)$ is said to be separation preserving if, for any $f$ and $g$ in $\mathrm{L}^{2}(\mu)$ with $f g=0 \mu$-almost everywhere, $(U f)(U g)=0$ also holds $\mu$-almost everywhere. A bounded linear operator $U$ is said to be positivity preserving if $U f \geqslant 0$, whenever $f \geqslant 0$. Any $C_{0}$-group $U_{t}(t \in \mathbb{R})$ of positivity preserving operators is also separation preserving (see $[\mathbf{1}$, p. 55]).

Proposition 5.2. Suppose that the unitary group $U_{t}=\mathrm{e}^{\mathrm{i} t A}$ has $U_{t}$ separation preserving for each $t \in \mathbb{R}$. Then for any $f \in \mathrm{L}^{2}(\mu)$ and $\delta>0$, the series $\sum_{n} a_{n} \varphi_{n}(x)$ is $(C, \delta)$ summable to $f(x)$ for $\mu$-almost all $x$.

Proof. The proof is based upon a transference technique, where we pass from the translation group $\tau_{u}: f(x) \mapsto f(x+u)$ on the line to the unitary group $U_{t}$ on $\mathrm{L}^{2}(\mu)$. By the spectral theorem, we can write, for any $f \in \mathrm{L}^{2}(\mu)$,

$$
(I-t L)_{+}^{\delta} f(x)=\frac{1}{2 \pi} \int_{-\infty}^{\infty} h_{\delta}(u) U_{t u} f(x) \mathrm{d} u \quad(x \in X),
$$

where $h_{\delta}$ is the Fourier transform of $(1-|x|)_{+}^{\delta}$. One can show that $\left|h_{\delta}(u)\right| \leqslant$ $\kappa_{\delta}\left(1+u^{2}\right)^{-(1+\delta) / 2}(u \in \mathbb{R})$ for some $\kappa_{\delta}<\infty$. The operator $(I-t L)_{+}^{\delta}$ may thus be obtained by transferring to $\mathrm{L}^{2}(\mu)$ the bounded convolution operator

$$
\int_{-\infty}^{\infty} h_{\delta}(u) \tau_{t u} \mathrm{~d} u
$$

on $\mathrm{L}^{2}(\mathbb{R})$; further, by Theorem 2.3 in $[\mathbf{1}]$, the maximal operator for the family $(I-t L)_{+}^{\delta}$ may be obtained by transference of the corresponding maximal convolution operator. To check that the latter is strongly $(2,2)$ bounded, we note that for any $g \in \mathrm{L}^{2}(\mathbb{R})$ we have

$$
\begin{aligned}
\sup _{t>0}\left|\int_{-\infty}^{\infty} h_{\delta}(u) \tau_{t u} g(x) \mathrm{d} u\right| & \leqslant \sup _{t>0} \kappa_{\delta} \int_{-\infty}^{\infty} \frac{1}{\left(1+u^{2}\right)^{(1+\delta) / 2}}|g(x+t u)| \mathrm{d} u \\
& \leqslant \sup _{t>0}(1+\delta) \kappa_{\delta} \int_{0}^{\infty} \frac{u^{2}}{\left(1+u^{2}\right)^{(\delta+3) / 2}} \frac{1}{u} \int_{-u}^{u}|g(x+t v)| \mathrm{d} v \mathrm{~d} u,
\end{aligned}
$$

where the last step follows from Fubini's Theorem. The latest integral is a convex combination of averages of $|g|$, and so is strongly $(2,2)$ bounded by the Hardy-Littlewood maximal averages theorem [15, p. 237]. By the transference theorem for maximal operators, $\sup _{t>0}(I-t L)_{+}^{\delta}$ is likewise strongly $(2,2)$ bounded, as required.

This proof of Proposition 5.2 ultimately reduced to the following observation.

Proposition 5.3. Suppose that $t \mapsto \mathrm{e}^{\mathrm{i} t A}$ is a bounded and strongly continuous representation of $\mathbb{R}$ on $\mathrm{L}^{p}(\mu)$, for some $1<p<\infty$, with each $\mathrm{e}^{\mathrm{i} t A}$ separation preserving; and let $\delta>0$. Then there exists $\kappa_{\delta}<\infty$ such that $C_{*}^{\delta} f \leqslant \kappa_{\delta} S_{*} f$ for all $f$; and $S_{*}$ is bounded $\mathrm{L}^{p}(\mu) \rightarrow \mathrm{L}^{p}(\mu)$. 


\section{Subordination of maximal functions}

Let $X$ be a complete Riemannian manifold with metric $\rho$ and measure $\mu$. We let $L$ be the Laplace operator in $\mathrm{L}^{2}(\mu)$, which is positive and self-adjoint. The wave operator $\cos (t \sqrt{L})$ has a distributional kernel $\cos (t \sqrt{L})(x, y)$ supported on the strip $\left\{(x, y) \in X^{2}\right.$ : $\rho(x, y) \leqslant t\}$ surrounding the diagonal $x=y$; thus, the solutions to the wave equation are of finite propagation speed [8]. In view of Remark $4.2(\mathrm{a})$, it is natural to regard the maximal function $W_{*}$ for the wave sine family $\sin (t \sqrt{L}) / t \sqrt{L}$ as the analogue of the Hardy-Littlewood maximal function.

The Poisson semigroup may be defined by

$$
P_{t} f(x)=\mathrm{e}^{-t \sqrt{L}} f(x)=\frac{1}{\pi} \int_{-\infty}^{\infty} \frac{t}{t^{2}+u^{2}} \cos (u \sqrt{L}) f(x) \mathrm{d} u
$$

for $f \in \mathrm{L}^{2}(\mu)$, and it extends to define a symmetric diffusion semigroup on $\mathrm{L}^{p}(\mu)$ for $1 \leqslant p \leqslant \infty[\mathbf{8}, \mathbf{1 8}]$. Here $V(t, x)=P_{t} f(x)$ defines a harmonic function, in the sense that $\left(\partial^{2} / \partial t^{2}-L\right) V(t, x)=0$ on $(0, \infty) \times X$. The maximal functions for the Poisson and heat semigroups are denoted $P_{*} f(x)=\sup _{t>0}\left|P_{t} f(x)\right|$ and $H_{*} f(x)=\sup _{t>0}\left|H_{t} f(x)\right|$, respectively. These may be compared pointwise in the following result.

Proposition 6.1. The maximal functions satisfy

(a) $P_{*} f(x) \leqslant H_{*} f(x) \leqslant W_{*} f(x)$ for all $f \in C_{\mathrm{c}}^{\infty}(X)$ and $x \in X$;

(b) $C_{*}^{\delta} f(x) \leqslant \kappa_{\delta} S_{*} f(x)$ for some constant $\kappa_{\delta}$ where $1<\delta<2$; and

(c) $S_{*} f(x) \leqslant \kappa P_{*} f(x)$, whenever $\cos (t \sqrt{L})(t>0)$ is positivity preserving.

Proof. The Poisson and heat semigroups $H_{t}=\mathrm{e}^{-t L}$ are related by the Bochner subordination formula [18, p. 47]

$$
P_{t} f(x)=\int_{0}^{\infty} \frac{H_{t^{2} / v} f(x) \mathrm{e}^{-v}}{\sqrt{v \pi}} \mathrm{d} v
$$

whereas one can obtain the relation

$$
H_{t} f(x)=\sqrt{\frac{2}{\pi}} \int_{0}^{\infty} s^{2} \mathrm{e}^{-s^{2} / 2} \frac{\sin (s \sqrt{2 t L})}{s \sqrt{2 t L}} f(x) \mathrm{d} s
$$

by integration by parts and Fourier inversion [8]. And so by convexity the maximal functions satisfy (a).

(b) We take $1<\delta<2$ and introduce $h_{\delta}(u)=\int_{-1}^{1}(1-|x|)^{\delta} \cos (u x) \mathrm{d} x$, which is a continuously differentiable and integrable function on $\mathbb{R}$. Using repeated partial integration, one shows that $\sigma_{\delta}(u)=-u h_{\delta}^{\prime}(u)$ is integrable over $\mathbb{R}$. By Fourier inversion and integration by parts, one obtains

$$
(1-t L)_{+}^{\delta}=\frac{1}{2 \pi} \int_{-\infty}^{\infty} \frac{\sin (u t L)}{u t L} \sigma_{\delta}(u) \mathrm{d} u
$$


from the spectral theorem. Now (b) follows by convexity, with

$$
\kappa_{\delta}=\frac{1}{2 \pi} \int_{-\infty}^{\infty}\left|\sigma_{\delta}(u)\right| \mathrm{d} u .
$$

(c) This follows from (4.8) and (6.1), since $F(u) \leqslant C /\left(1+u^{2}\right)$.

Remarks 6.2. (1) On account of (4.1), the hypothesis of Proposition 6.1 (b) is satisfied by the Euclidean Laplace operator.

(2) The inequality Proposition 6.1 (b) is the basis for Hardy's theorem on the almost everywhere summability of classical Fourier series [12, Theorem 3] over $\mathbb{T}$; the method of Kolmogorov-Seliverstov is rather different in that it involves detailed analysis of kernel functions $[\mathbf{1 4}]$.

(3) Results concerning spherical sums of classical Fourier series over $\mathbb{T}^{d}$ with $p \neq 2$ are sensitive to dimension. Carleson and Sjölin showed that the operators $(I-t \Delta)_{+}^{\delta}$ are bounded $\mathrm{L}^{p}\left(\mathbb{R}^{2}\right) \rightarrow \mathrm{L}^{p}\left(\mathbb{R}^{2}\right)$ for $\frac{4}{3} \leqslant p \leqslant 4$ for $t, \delta>0$. Using our method with [5, Lemma 3.1] and (5.2), one obtains the weaker result that the $(I-t \Delta)_{+}^{\delta}(t>0)$ are uniformly bounded $\mathrm{L}^{p}\left(\mathbb{R}^{2}\right) \rightarrow \mathrm{L}^{p}\left(\mathbb{R}^{2}\right)$ for $4 /(2+\delta)<p<4 /(2-\delta)$. By contrast, $\delta=0$ gives the multiplier for the disc, which is famously unbounded on $\mathrm{L}^{p}\left(\mathbb{R}^{2}\right)$ for $p \neq 2[\mathbf{1 1}]$.

(4) In the analysis of harmonic functions on the disc, the non-tangential maximal function is often used in conjunction with square functions such as the LittlewoodPaley $g$-function. In $[\mathbf{1 0}]$ it is shown that an operator with a bounded $\mathrm{H}^{\infty}$ functional calculus on $\mathrm{L}^{p}$ satisfies square function estimates. It would be interesting to determine how such bounds are related to maximal-operator bounds, and whether the maximaloperator bounds can be dualized.

Acknowledgements. I thank G. J. O. Jameson, T. A. Gillespie and E. B. Davies for helpful remarks, and the referee for pointing out reference [10].

\section{References}

1. N. Asmar, E. Berkson and T. A. Gillespie, Transference of strong type maximal inequalities by separation-preserving representations, Am. J. Math. 113 (1991), 47-74.

2. E. BERKSON AND T. A. GILlespie, Spectral decompositions and harmonic analysis on UMD spaces, Studia Math. 112 (1994), 13-49.

3. E. Berkson, T. A. Gillespie And P. S. Muhly, Abstract spectral decompositions guaranteed by the Hilbert transform, Proc. Lond. Math. Soc. (3) 53 (1986), 489-517.

4. G. Blower, Multipliers for semigroups, Proc. Edinb. Math. Soc. 39 (1996), 241-252.

5. G. Blower, Maximal functions for groups of operators, Proc. Edinb. Math. Soc. 43 (2000), 57-71.

6. J. Bourgain, Some remarks on Banach spaces in which martingale difference sequences are unconditional, Ark. Mat. 21 (1983), 163-168.

7. J. Bourgain, Vector-valued singular integrals and the $H^{1}-B M O$ duality, in Probability theory and harmonic analysis (ed. J.-A. Chao and W. A. Woyczyński), pp. 1-19 (Marcel Dekker, New York, 1986).

8. J. Cheeger, M. Gromov and M. TAylor, Finite propagation speed, kernel estimates for functions of the Laplace operator, and the geometry of complete Riemannian manifolds, J. Diff. Geom. 17 (1982), 15-53. 
9. M. G. Cowling, Harmonic analysis on semigroups, Ann. Math. 117 (1983), 267-283.

10. M. G. Cowling, I. Doust, A. Mcintosh And A. Yagi, Banach space operators with a bounded $H^{\infty}$ functional calculus, J. Aust. Math. Soc. A 60 (1996), 51-89.

11. C. Fefferman, The multiplier problem for the ball, Ann. Math. 94 (1971), 330-336.

12. G. H. Hardy, On the summability of Fourier's series, Proc. Lond. Math Soc. (2) 12 (1913), 365-372.

13. C. E. Kenig And A. Ruiz, A strong $(2,2)$ estimate for a maximal operator associated to the Schrödinger operator, Trans. Am. Math. Soc. 280 (1983), 239-246.

14. A. N. Kolmogorov and C. R. Seliverstov, On convergence of Fourier series, $C . R$. Acad. Sci. Paris Ser. 1178 (1924), 303-306.

15. P. Koosis, Introduction to $H_{p}$ spaces (Cambridge University Press, 1980).

16. D. Menshov, Sur les series de fonctions orthogonales, Fund. Math. 4 (1928), 82-105.

17. A. Moyua, A. VArgas AND L. VEGA, Schrödinger maximal function and restriction properties of the Fourier transform, Int. Math. Res. Not. 16 (1996), 793-815.

18. E. M. Stein, Topics in harmonic analysis related to the Littlewood-Paley theory, Annals of Mathematical Studies, vol. 63 (Princeton University Press, Princeton, NJ, 1970).

19. E. M. Stein, Harmonic analysis: real variable methods, orthogonality and oscillatory integrals (Princeton University Press, Princeton, NJ 1993).

20. E. C. Titchmarsh, The theory of functions, 2nd edn (Oxford University Press, 1938)

21. E. C. Titchmarsh, Introduction to the theory of Fourier integrals, 2nd edn (Oxford University Press, 1948). 